\title{
Electric Potential and Field Near Pointed Shaped Surfaces
}

\author{
C.F.A. Souza, N.P. Andion and C.M.C. de Castilho \\ Instituto de Física, Universidade Federal da Bahia, Campus Universitário da Federação, \\ 40210-340 Salvador, Bahia, Brazil
}

\begin{abstract}
The trajectories of charged particles, emitted from within or from the close vicinity of pointed shaped surfaces, requires the knowledge of the electric field resulting from the potential bias between surface and detector, or screen. Frequently it is necessary the use of numerical methods for solving Laplace's equation as a result of difficulties in obtaining an analytical expression. Recently we have shown that, when any two coordinate surfaces of an orthogonal system are kept at two different but constant potentials, it is possible to obtain an analytical solution for the potential in a relatively simple manner. Using this general property of orthogonal coordinate systems, we present the solution for the electric potential and field in the vicinity of pointed surfaces for several cases of practical interest in field emission, field ionisation, atomprobe field ion spectroscopy and related phenomena.
\end{abstract}

\section{INTRODUCTION}

The adequate modelling of the surface geometry, electric potential and field in the region close to the sample in the Field Ion Microscope (FIM) and in the Field Emission Microscope (FEM) is an important procedure in determining quantities related to tunnelling probabilities as well as the trajectories of the charged particles, ions or electrons. To properly deal with this we have to consider two different aspects: i) the overall variation of field and potential as a result of the general shape of the sample, regardless the minor protrusions present in the sample's surface when treating the problem in an atomic scale; ii) the local variation of field and potential which results exactly from the atomic scale structure just mentioned. In this work we restrict ourselves in considering just the first case. We present an analytical solution of Laplace's equation in a region bounded by two surfaces held at constant but different potentials, i. e., in a region with boundary conditions such that we have a simple case of the Dirichlet problem $/ 1 /$. We also discuss a few cases of practical interest, determining the ratio between field and potential at special points of the conductors. These special cases, are disperse in the literature $/ 2,3 /$, presenting sometimes conflict between them, and not always determined under the same approach as we do here.

For solving partial differential equations, the general methods available are the integral solution and the separated solution $/ 2 /$. However, for a certain class of geometries, we can show that Laplace's solutions can be obtained from a much faster and straightforward method. We call it the single-valued Laplace's solution method and describe it as follows. For an orthogonal system of coordinates $\left(q_{1}, q_{2}, q_{3}\right.$ and unit vectors $\mathbf{e}_{2}, \mathbf{e}_{2}, \mathbf{e}_{3}$ ) with metric coefficients $h_{1}, h_{2}, h_{3}$ (also known as scale factor $/ 2 /$ and metric tensor since it is a second-rank tensor /4/), Laplace's equation can be written as: 
$\nabla^{2} \mathrm{~V}=\left(1 / \mathrm{H}^{3}\right) \sum_{\mathrm{i}=1}^{3}\left(\partial / \partial \mathrm{q}_{\mathrm{i}}\right)\left[\left(\mathrm{H}^{3} / \mathrm{h}_{\mathrm{i}}{ }^{2}\right)\left(\partial \mathrm{V} / \partial \mathrm{q}_{\mathrm{i}}\right)\right]=0$

where $\mathrm{V}$ is the electric potential and

$H^{3}=h_{1} h_{2} h_{3}$

A coordinate surface for such a system corresponds to the loci of points where one of the coordinates is constant.

It can be shown 15/ that, for the set of orthogonal systems listed in Table 1, the solution of Laplace's equation in the region bounded by two coordinate surfaces, both related to the same coordinate, say $q_{1}$; can be written as a function of just this coordinate. In a mathematical language this corresponds to state that, if

$\mathrm{V}=\alpha$ for $\mathrm{q}_{1}=\mathrm{a}, \forall \mathrm{q}_{2}$ and $\mathrm{q}_{3}$;

$\mathrm{V}=\boldsymbol{\beta}$ for $\mathrm{q}_{1}=\mathrm{b}, \forall \mathrm{q}_{2}$ and $\mathrm{q}_{3}$

with $\alpha \neq \beta$, and assuming also that it is possible to write

$\mathrm{H}^{3} / \mathrm{h}_{1}^{2}=\mathrm{h}_{2} \mathrm{~h}_{3} / \mathrm{h}_{1}=\mathrm{f}\left(\mathrm{q}_{1}\right) \mathrm{g}\left(\mathrm{q}_{2}, \mathrm{q}_{3}\right)$,

then the solution of Laplace's equation, $V$, depends only on $q_{1}$ and has the form

$\mathrm{V}=\mathrm{A} \int\left[1 / \mathrm{f}\left(\mathrm{q}_{1}\right)\right] \mathrm{d} \mathrm{q}_{1}+\mathrm{B}$

where $\mathrm{A}$ and $\mathrm{B}$ are constants which are determined from the boundary conditions expressed by eqs. 3 .

From the general expression for the gradient of $\mathrm{V}$,

$\operatorname{grad} \mathrm{V}=\left(1 / \mathrm{h}_{1}\right)\left(\partial \mathrm{V} / \partial \mathrm{q}_{1}\right) \mathbf{e}_{1}+\left(1 / \mathrm{h}_{2}\right)\left(\partial \mathrm{V} / \partial \mathrm{q}_{2}\right) \mathbf{e}_{2}+\left(1 / \mathrm{h}_{3}\right)\left(\partial \mathrm{V} / \partial \mathrm{q}_{3}\right) \mathbf{e}_{3}$

it is possible to express the electric field at the region bounded by two coordinate surfaces (conditions 3 , a and $b$ ) as:

$\mathbf{E}=-\mathrm{A} /\left[\mathrm{h}_{1} \mathrm{f}\left(\mathrm{q}_{1}\right)\right] \mathbf{e}_{\mathrm{k}}$

The condition expressed by eq. 4 restricts the set of possible orthogonal systems for which a solution with the form of eq. 5 can be found and so the shape of the coordinate surfaces. In table 1 it is shown the possible geometries for coordinate surfaces of the various systems of coordinates we have considered.

Table 1 - Correspondence between coordinate surfaces and kind of geometry.

\begin{tabular}{|c|c|c|}
\hline Coordinate systems & Coordinates & $\begin{array}{l}\text { Coordinate Surfaces } \\
\text { (corresponding number) * }\end{array}$ \\
\hline Cartesian & \begin{tabular}{|lll}
$x$ & $y$ & $z$
\end{tabular} & $\begin{array}{lll}1 & 1 & 1 \\
\end{array}$ \\
\hline Spherical & $\begin{array}{lll}\mathbf{r} & \theta & \phi\end{array}$ & $\begin{array}{lll}2 & 3 & 4\end{array}$ \\
\hline Circular cylinder & $\rho \phi z$ & 544 \\
\hline Elliptic cylinder & $\mu \phi z$ & $\begin{array}{lll}6 & 7 & 1 \\
\end{array}$ \\
\hline Parabolic cylinder & $\mu \quad v z$ & \begin{tabular}{|lll}
8 & 8 & 1 \\
\end{tabular} \\
\hline Parabolic (rotational) & $\lambda \mu \phi$ & $\begin{array}{lll}9 & 9 & 4 \\
\end{array}$ \\
\hline Paraboloidal & $\xi_{1} \xi_{2} \xi_{3}$ & \begin{tabular}{|l|l|l|}
10 & 11 & 10 \\
\end{tabular} \\
\hline Oblate spheroidal & $\mu \theta \phi$ & $\begin{array}{|lll|}12 & 13 & 4\end{array}$ \\
\hline Prolate spheroidal & $\mu \theta \phi$ & \begin{tabular}{|l|l|l}
14 & 15 & 4
\end{tabular} \\
\hline Conical & $\xi_{2} \xi_{2} \xi_{3}$ & $\begin{array}{lll}2 \quad 16 \quad 16 \\
\end{array}$ \\
\hline Ellipsoidal & $\xi_{1} \xi_{2} \xi_{3}$ & \begin{tabular}{|lll}
17 & 18 & 19 \\
\end{tabular} \\
\hline
\end{tabular}

* Each number above corresponds to a specific coordinate surface as listed below:

1. parallel planes; 2 concentric spheres; 3 . circular cones of one sheet; 4 . axial planes; 5 . concentric cylinders; 6 . confocal elliptic cylinders; 7. hyperbolic cylinders; 8. confocal parabolic cylinders; 9. paraboloids of revolution; 10. elliptic paraboloids; 11. hyperbolic paraboloids; 12 . oblate spheroids; 13. hyperboloids of revolution (1 sheet); 14. prolate spheroids ; 15. kyperboloids of revolution (2 sheets); 16. elliptic cones 17.ellipsoids; 18. hyperboloids of 1 sheet; 19. hyperboloids of 2 sheets. 
Table 2 - Expressions for electric field and potential for each kind of geometry of table 1 (bold-faced and non-italic letters).

\begin{tabular}{|c|c|c|}
\hline Geometry & Potential & Field \\
\hline $\begin{array}{r}\text { parallel planes } \\
\text { (1) }\end{array}$ & $V=A x+B$ & $\mathbf{E}=-\mathrm{A} \mathbf{e}_{\mathbf{x}}$ \\
\hline $\begin{array}{ll}\text { concentric } \\
\text { spheres }\end{array}$ & $V=A / r+B$ & $E=A / r^{2} \mathbf{e}_{r}$ \\
\hline $\begin{array}{l}\text { cones of one } \\
\text { sheet }\end{array}$ & $\mathrm{V}=\mathrm{A} \ln |\tan (\theta / 2)|+\mathrm{B}$ & $E=-A /[r \sin (\theta)] e_{\theta}$ \\
\hline axial planes (4) & $V=A \phi+B$ & $\mathbf{E}=-\mathbf{A} /[\mathrm{r} \sin (\theta)] \mathbf{e}_{\phi}$ \\
\hline $\begin{array}{ll}\begin{array}{l}\text { concentric } \\
\text { cylinders }\end{array} \quad \text { (5) } \\
\end{array}$ & $V=A \ln (\rho)+B$ & $\mathbf{E}=-\mathbf{A} / \rho \mathbf{e}_{\boldsymbol{\rho}}$ \\
\hline $\begin{array}{l}\text { confocal elliptic } \\
\text { cylinders } \quad \text { (6) }\end{array}$ & $V=A \mu+B$ & $E=-A /\left[d\left(\cosh ^{2} \mu-\cos ^{2} \phi\right)^{1 / 2}\right] \mathbf{e}_{\mu}$ \\
\hline $\begin{array}{l}\text { hyperbolic } \\
\text { cylinders } \quad(7)\end{array}$ & $\mathrm{V}=\mathrm{A} \phi+\mathrm{B}$ & $\mathbf{E}=-\mathrm{A} /\left[\mathrm{d}\left(\cosh ^{2} \mu-\cos ^{2} \phi\right)^{1 / 2}\right] \mathbf{e}_{\phi}$ \\
\hline $\begin{array}{|ll|}\text { confocal } & \\
\text { parabolic } & \\
\text { cylinders } & (8) \\
\end{array}$ & $V=A \mu+B$ & $\mathbf{E}=-\mathbf{A} /\left[2 \mathrm{D}\left(\mu^{2}+v^{2}\right)^{1 / 2}\right] \mathbf{e}_{\boldsymbol{\mu}}$ \\
\hline $\begin{array}{l}\text { paraboloids of } \\
\text { revolution } \quad(9)\end{array}$ & $V=A \ln (\lambda)+B$ & $\mathbf{E}=-\mathbf{A} /\left[2 \mathrm{D} \lambda\left(\lambda^{2}+\mu^{2}\right)^{1 / 2}\right] \mathbf{e}_{\lambda}$ \\
\hline $\begin{array}{l}\text { elliptic } \\
\text { paraboloids } \\
\text { (10) }\end{array}$ & $\mathrm{V}=\mathrm{A} \ln \left|\left(\xi_{1}{ }^{2}-\mathrm{a}^{2}\right)^{1 / 2}+\left(\xi_{1}{ }^{2}-\mathrm{b}^{2}\right)^{1 / 2}\right|+\mathrm{B}$ & $\mathbf{E}=-\mathrm{A} /\left[\left(\xi_{1}{ }^{2}-\xi_{2}{ }^{2}\right)\left(\xi_{1}{ }^{2}-\xi_{3}{ }^{2}\right)\right]^{1 / 2} \mathbf{e}_{\boldsymbol{\xi} 1}$ \\
\hline \begin{tabular}{|l|} 
hyperbolic \\
paraboloids \\
(11)
\end{tabular} & $V=A \ln \left|\left(\xi_{2}{ }^{2}-a^{2}\right)^{1 / 2}+\left(\xi_{2}{ }^{2}-b^{2}\right)^{1 / 2}\right|+B$ & $\mathbf{E}=-\mathbf{A} /\left[\left(\xi_{2}{ }^{2}-\xi_{1}{ }^{2}\right)\left(\xi_{2}{ }^{2}-\xi_{3}{ }^{2}\right)\right]^{1 / 2} \mathbf{e}_{\xi_{2}}$ \\
\hline $\begin{array}{l}\text { oblate } \\
\text { spheroids (12) }\end{array}$ & $V=A \tan ^{-1}[\tanh (\mu / 2)]+B$ & $\mathbf{E}=-\mathrm{A} /\left[2 \mathrm{~d} \cosh (\mu)\left[\cosh ^{2}(\mu)-\cos ^{2}(\theta)\right]^{1 / 2}\right] \mathbf{e}_{\mu}$ \\
\hline $\begin{array}{l}\text { hyperboloids of } \\
\text { revolution of } 1 \\
\text { sheet } \quad(13)\end{array}$ & $\mathrm{V}=\mathrm{A} \ln |[1+\tan (\theta / 2)] /[1-\tan (\theta / 2)]|+\mathrm{B}$ & $\mathbf{E}=-2 \mathrm{~A} /\left[\mathrm{d} \cos (\theta)\left[\cosh ^{2}(\mu)-\cos ^{2}(\theta)\right]^{1 / 2}\right] \mathbf{e}_{\theta}$ \\
\hline \begin{tabular}{|l|} 
prolate \\
spheroids (14)
\end{tabular} & $\mathrm{V}=\mathrm{A} \ln |\tanh (\mu / 2)|+\mathrm{B}$ & $\mathbf{E}=-\mathrm{A} /\left[\mathrm{d} \sinh (\mu)\left[\cosh ^{2}(\mu)-\cos ^{2}(\theta)\right]^{1 / 2}\right] \mathbf{e}_{\mu}$ \\
\hline $\begin{array}{l}\text { hyperboloids of } \\
\text { revolution of } 2 \\
\text { sheets (15) }\end{array}$ & $\mathrm{V}=\mathrm{A} \ln |\tan (\theta / 2)|+\mathrm{B}$ & $\left.\mathbf{E}=-\mathrm{A} /\left[\mathrm{d} \sin (\theta)\left[\cosh ^{2}(\mu)-\cos ^{2}(\theta)\right]\right]^{1 / 2}\right] \mathbf{e}_{\theta}$ \\
\hline cones* & elliptic integral & $\mathbf{E}=-\mathbf{A} /\left[\xi_{1}\left(\xi_{2}{ }^{2}+\xi_{3}{ }^{2}\right)^{1 / 2}\right] \mathbf{e}_{\xi_{2}}$ \\
\hline ellipsoids (17) & elliptic integral & $\mathbf{E}=-\mathbf{A} /\left[\left(\xi_{1}{ }^{2}-\xi_{2}{ }^{2}\right)\left(\xi_{1}{ }^{2}-\xi_{3}{ }^{2}\right)\right]^{1 / 2} \mathbf{e}_{\xi_{1}}$ \\
\hline $\begin{array}{l}\text { hyperboloids of } \\
1 \text { sheet* } \quad \text { (18) }\end{array}$ & elliptic integral & $\mathbf{E}=-\mathrm{A} /\left[\left(\xi_{2}{ }^{2}-\xi_{1}{ }^{2}\right)\left(\xi_{2}{ }^{2}-\xi_{3}{ }^{2}\right)\right]^{1 / 2} \mathbf{e}_{\xi_{2}}$ \\
\hline $\begin{array}{l}\text { hyperboloids of } \\
2 \text { sheets* }\end{array}$ & elliptic integral & $\mathbf{E}=-\mathrm{A} /\left[\left(\xi_{3}{ }^{2}-\xi_{1}{ }^{2}\right)\left(\xi_{3}{ }^{2}-\xi_{2}{ }^{2}\right)\right]^{1 / 2} \mathbf{e}_{\xi_{3}}$ \\
\hline
\end{tabular}

*elliptic

\section{CASES OF PRACTICAL INTEREST}

From a total of thirty three coordinates in table 1, only nineteen correspond to distinct geometries. From these, four cases do not present an analytical solution since it would be necessary to solve elliptical integrals. The other fifteen have an analytical solution and Table 2 presents the general form of the expressions for the electric potential and field.

From the fifteen cases above mentioned, six can be considered as being of practical interest: concentric spheres, circular cones (one sheet), concentric cylinders, paraboloids of revolution, prolate spheroids and hyperboloids of revolution ( 2 sheets). Frequently, for emitters or other sharpened conductors, it is possible to measure the electric potential with great accuracy $(0.01 \%$ or even less) but the field determination 
depends on geometric characteristics of the sample which are expressed by the field proportionality factor, i. e, the ratio between field $\left(F_{0}\right)$ and potential $\left(V_{0}\right)$ at close vicinity of the apex of a conductor with sharp geometry. It is then expressed by

$\beta=\mathrm{F}_{\alpha} / \mathrm{V}_{\mathrm{o}}$

Several authors have determined the value of $\beta$ for various geometries $/ 6-10 /$, with or without approximations, getting sometimes slightly different results as a consequence of the referential for the electric potential. We present values of $\beta$ for a few cases of interest in table 3 .

Table 3 - Field proportionality factor for some geometries of practical interest $\left(\beta=F_{0} / V_{0}\right)$

\begin{tabular}{|l|l|c|}
\hline geometry & $\beta$ (exact) & $\beta$ (approximation) \\
\hline spherical & $1 / \mathbf{r}_{t}$ & - \\
\hline $\begin{array}{l}\text { concentric } \\
\text { cylinders }\end{array}$ & $1 /\left[\mathrm{r}_{\mathrm{o}} \ln \left(\mathrm{R} / \mathrm{r}_{\mathrm{o}}\right)\right]$ & - \\
\hline hyperboloid & $4 \mathrm{~d} /\left[\left[-\mathrm{r}_{\mathrm{t}}{ }^{2} \pm \mathrm{r}_{\mathrm{t}}\left(\mathrm{r}_{\mathrm{t}}{ }^{2}+4 \mathrm{~d}^{2}\right)^{1 / 2}\right]\left[\ln \left[\left(\left(2 \mathrm{~d}+\left(\mathrm{r}_{\mathrm{t}}{ }^{2}+4 \mathrm{~d}^{2}\right)^{1 / 2}\right) / \mathbf{r}_{\mathrm{t}}\right)^{ \pm 1}\right]\right]\right]$ & $1 /\left[\mathrm{r}_{\mathrm{t}} \ln \left(2\left(\mathrm{~d} / \mathrm{r}_{\mathrm{t}}\right)^{1 / 2}\right)\right]$ \\
\hline paraboloid & $2 /\left[\mathrm{r}_{\mathrm{t}} \ln \left(2 \mathrm{D} / \mathrm{r}_{\mathrm{t}}\right)\right]$ & - \\
\hline prolate spheroid & $\left.4 \mathrm{~d} /\left[\left[\mathrm{r}_{\mathrm{t}}{ }^{2} \pm \mathrm{r}_{\mathrm{t}}\left(\mathrm{r}_{\mathrm{t}}{ }^{2}+4 \mathrm{~d}^{2}\right)^{1 / 2}\right]\left[\ln \mid\left(\left(2 \mathrm{~d}+\left(\mathrm{r}_{\mathrm{t}}{ }^{2}+4 \mathrm{~d}^{2}\right)^{1 / 2}\right) / \mathrm{r}_{\mathrm{t}}\right)^{ \pm 1}\right]\right]\right]$ & $1 /\left[\mathrm{r}_{\mathrm{t}} \ln \left(2\left(\mathrm{~d} / \mathrm{r}_{\mathrm{t}}\right)^{1 / 2}\right)\right]$ \\
\hline conical & diverges & diverges \\
\hline
\end{tabular}

$$
\begin{aligned}
& \mathrm{r}_{\mathrm{t}}=\text { radius of curvature; } \\
& \mathrm{R}=\text { external radius of the cylinder; } \\
& \mathrm{d}=\text { focal distance ( ellipse/hyperbola ); } \\
& \mathrm{D}=\text { constant } \\
& \mathrm{r}_{0}=\text { internal radius of the cylinder }
\end{aligned}
$$

\section{FINAL COMMENTS}

We have determined the expressions for the field and electric potential in several regions where it is possible to solve Laplace's equation by adopting as boundary conditions a constant potential at each one of two different coordinate surfaces. This has been done using a general theorem where the basic requirement is that expressed by eq. 4 . The ratio between field and potential at the vicinity of the apex of a few sharped geometries was then calculated.

\section{ACKNOWLEDGEMENT}

We would like to acknowledge the collaboration of Miss P. M. Costa during the first part of this work. This work has financial support of UFBA and CNPq (Brazil)

\section{REFERENCES}

1. Jackson, J. D., Classical Electrodynamics ( John Wiley \& Sons, New York, 1962).

2. Morse, P. M. and Feshbach, H., Methods of Theoretical Physics (McGraw-Hill, New York, 1953).

3. Schelkunoff, S. A., Applied Mathematics for Engineers and Scientists (D. van Nostrand, Princeton, 1965).

4. Arfken, G. , Mathematical Methods for Physicists (Academic Press, New York, 1985)

5. Andion, N. P. and de Castilho, C. M. C., to be published.

6. Sakurai, T. and Müller, E. W., Jrnl. of Applied Physics, 48, 2618, 1977.

7. Beckey, H. D., Krone, H. and Roellgen, F. W., Jrnl. of Sci. Instr. 1, 118, 1968.

8. Birdseye, P. J. and Smith, D. A., Surf. Sci. 23,198, 1970.

9. Coelho, R. and Debeau, J., J. Phys. D: Appl. Phys. 4, 1266, 1971.

10. de Castilho and Kingham, Surf. Sci., 173, 75, 1986. 